\title{
Analytical Modeling and Experimental Validation of NB-IoT Device Energy Consumption
}

\author{
Pilar Andres-Maldonado ${ }^{(}$, Mads Lauridsen ${ }^{(}$, , Pablo Ameigeiras $\left.^{(}\right)$, and Juan M. Lopez-Soler ${ }^{(}$
}

\begin{abstract}
The recent standardization of 3GPP Narrowband Internet of Things (NB-IoT) paves the way to support low-power wide-area (LPWA) use cases in cellular networks. NB-IoT design goals are extended coverage, low power and low cost devices, and massive connections. As a new radio access technology, it is necessary to analyze the possibilities NB-IoT provides to support different traffic and coverage needs. In this paper, we propose and validate an NB-IoT energy consumption model. The analytical model is based on a Markov chain. For the validation, an experimental setup is used to measure the energy consumption of two commercial NB-IoT user equipments (UEs) connected to a base station emulator. The evaluation is done considering three test cases. The comparison of the model and measurements is done in terms of the estimated battery lifetime and the latency needed to finish the control plane procedure. The conducted evaluation shows the analytical model performs well, obtaining a maximum relative error of the battery lifetime estimation between the model and the measurements of $21 \%$ for an assumed interarrival time (IAT) of $6 \mathrm{~min}$.
\end{abstract}

Index Terms-Analytical model, control plane (CP), energy consumption, latency, Narrowband Internet of Things (NB-IoT).

\section{INTRODUCTION}

A WIRELESS Internet of Things (IoT) radio access network has four conflicting key performance indicators (KPIs): 1) cost; 2) battery lifetime; 3) coverage; and 4) capacity. Traditional cellular networks fall short on meeting all of the four KPIs. Within this context, the third generation partnership project (3GPP) introduced a new access technology called Narrowband IoT (NB-IoT) in June 2016. NB-IoT is a set of

Manuscript received December 14, 2018; revised February 9, 2019 and February 21, 2019; accepted March 10, 2019. Date of publication March 13, 2019; date of current version June 19, 2019. This work was supported in part by the Spanish Ministry of Economy and Competitiveness and the European Regional Development Fund under Project TEC2016-76795-C6-4-R and in part by the H2020 European Project TRIANGLE under Grant 688712. (Corresponding author: Pilar AndresMaldonado.)

P. Andres-Maldonado was with the Wireless Communication Networks Section, Aalborg University, DK-9220 Aalborg, Denmark. She is now with the Research Center on Information and Communication Technologies, University of Granada, 18071 Granada, Spain, and also with the Department of Signal Theory, Telematics and Communications, University of Granada, 18071 Granada, Spain (e-mail: pilaram@ugr.es).

M. Lauridsen was with the Wireless Communication Networks Section, Aalborg University, DK-9220 Aalborg, Denmark. He is now with the Standardization Research Lab, Nokia Bell Labs, DK-9220 Aalborg, Denmark (e-mail: mads.lauridsen@nokia-bell-labs.com).

P. Ameigeiras and J. M. Lopez-Soler are with the Research Center on Information and Communication Technologies, University of Granada, 18071 Granada, Spain, and also with the Department of Signal Theory, Telematics and Communications, University of Granada, 18071 Granada, Spain (e-mail: pameigeiras@ugr.es; juanma@ugr.es).

Digital Object Identifier 10.1109/JIOT.2019.2904802 specifications particularly well fitted to the low-power widearea (LPWA) segment. NB-IoT was introduced in Release 13 and its design goals were [1].

1) Maximum latency of $10 \mathrm{~s}$ on the uplink (UL).

2) Target coverage of $164 \mathrm{~dB}$ maximum coupling loss (MCL).

3) User equipment (UE) battery lifetime beyond ten years, assuming a stored energy capacity of $5 \mathrm{Wh}$.

4) Massive connection density of 1000000 devices per square $\mathrm{km}$ in an urban environment.

Providing the coverage extension while maintaining low energy consumption is an indispensable characteristic of NB-IoT. However, this comprises a great challenge as the coverage extension is mainly achieved by trading off data rate, e.g., lowering the transmission bandwidth, or using repetitions in time. Additionally, the large range of possible configurations of the radio interface results in a significant variability of the UE performance. In order to satisfy the requirements of an IoT application, it is important to have a clear view of the possibilities NB-IoT provides.

In the current literature, [2] and [3] show the performance of the NB-IoT radio channels. Feltrin et al. [4] discussed the main sources of latency and present an evaluation of the resource occupation in different IoT cases. Azari et al. [5] proposed an analytical model based on queuing theory for the channel scheduling in NB-IoT. An NB-IoT and long term evolution (LTE) Cat-M1 simulator is presented in [6]. The simulator is based on the existing LTE module in NS-3 and evaluates the power consumption, scalability, and end-to-end latency. Furthermore, Lauridsen et al. [7] presented empirical power consumption measurements of two NB-IoT UEs.

The NB-IoT networks are eventually ready to roll-out in practical deployments. However, there is still unawareness if NB-IoT will be able to cope with the IoT KPIs due to the vast scenarios and configurations to consider. To address this issue, this paper proposes an analytical model for NB-IoT. This model provides an easy methodology for final users and operators to predict and plan if the desired IoT KPIs will be satisfied in terms of the expected UE battery lifetime and latency, and to estimate the impact of different configurations in the UE performance.

In our previous work [8], we proposed a preliminary model of energy consumption for LTE. This paper improves the energy consumption model used in [8] and adapts the analysis to include specific NB-IoT features. Additionally, this paper validates our NB-IoT model through empirical power consumption measurements. 
TABLE I

ACRONYMS LIST

\begin{tabular}{c|l}
\hline Acronym & \multicolumn{1}{|c}{ Description } \\
\hline CP & Control Plane optimization \\
DCI & Downlink Control Information \\
DL & Downlink \\
DRX & Discontinuous Reception \\
DUT & Device Under Test \\
IAT & Inter-Arrival Time \\
MCS & Modulation and Coding Scheme \\
NPDCCH & Narrowband Physical Downlink Control CHannel \\
NPDSCH & Narrowband Physical Downlink Shared CHannel \\
NPRACH & Narrowband Physical Random Access CHannel \\
NPUSCH & Narrowband Physical Uplink Shared CHannel \\
PSM & Power Saving Mode \\
RA & Random Access \\
RRC & Radio Resource Control \\
RU & Resource Unit \\
SF & Subframe \\
TBS & Transport Block Size \\
UE & User Equipment \\
\hline
\end{tabular}

Specifically, the main contributions of this paper are as follows.

1) Derivation of a tractable energy consumption and delay model for NB-IoT.

2) Inclusion of several NB-IoT unique aspects in the model that increase its accuracy, such as radio channels scheduling/allocation and hybrid automatic repeat request (HARQ) acknowledgments (ACKs).

3) Experimental validation of the model based on empirical energy consumption measurements. The experimental validation has considered three test cases and two commercial device under test (DUT).

The experimental setup used in this paper is similar to the one used in [7]. The empirical energy consumption measurements are obtained through a DUT connected to an NB-IoT base station emulator and a power analyzer. The comparison of the model and measurements is done in terms of the estimated battery lifetime and the latency needed to finish the control plane cellular IoT evolved packet system optimization (CP) procedure. The results show the analytical model performs well, obtaining a maximum relative error of the battery lifetime estimation between the model and the measurements of $21 \%$ for an assumed IAT of $6 \mathrm{~min}$. Additionally, from the results we claim that the NB-IoT UEs achieve both targets of ten years of battery lifetime and $10 \mathrm{~s}$ of latency for a large range of different setups when the traffic profile has a large IAT, or the radio resource configuration does not require an extensive number of repetitions.

The remainder of this paper is structured as follows. Section II describes the system model. Section III explains the key NB-IoT aspects considered in the model. Section IV details the proposed NB-IoT energy consumption model. Section $\mathrm{V}$ presents the experimental setup used in the validation. Section VI presents the validation with numerical results. Finally, Section VII sums up the conclusions. Table I lists the main acronyms used throughout this paper.

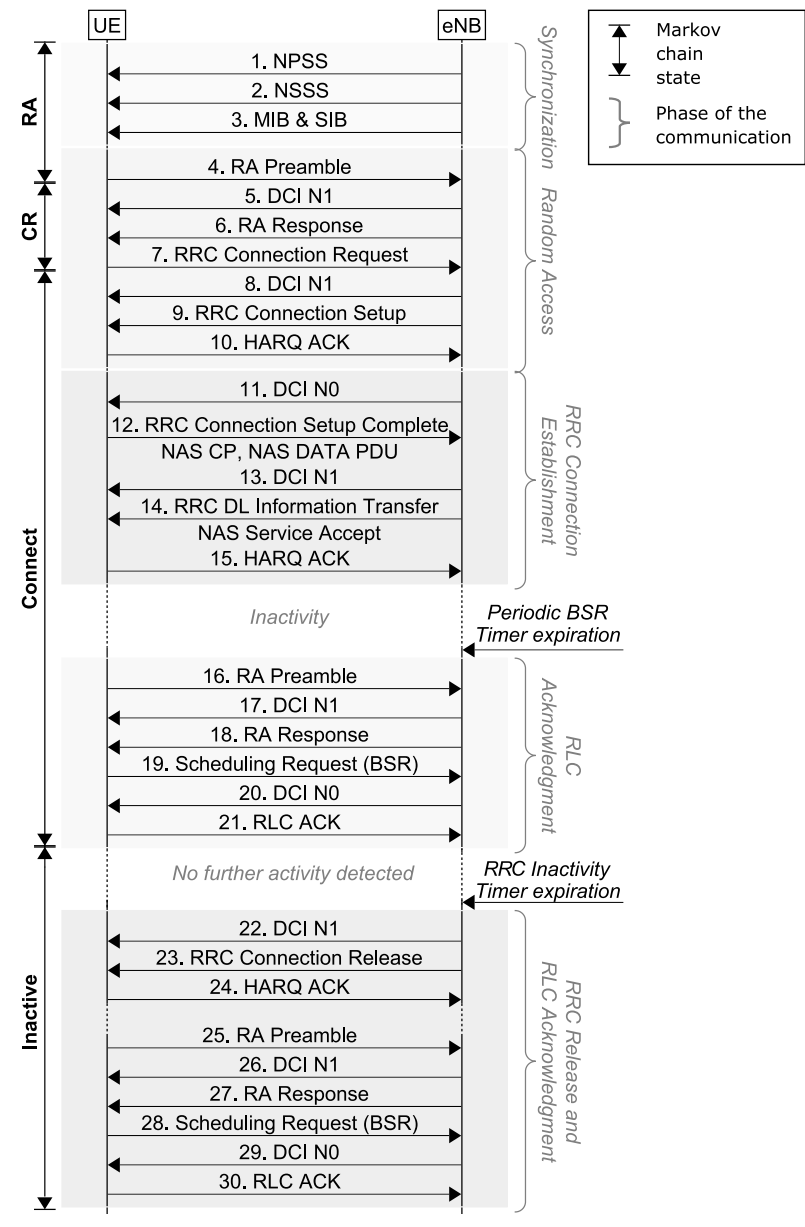

Fig. 1. Mobile originated data transport in CP [9].

\section{SySTEM MOdeL}

Assume a cell with an evolved NodeB (eNB) with an NB-IoT carrier deployed in-band, and one UE camping on it. The UE transfers UL reports of size $L$ periodically to the eNB. These reports are destined to an IoT server. We assume there is no ACK from the IoT server following the UL report. To send these periodic UL reports, the UE performs the CP procedure [9]. For NB-IoT UEs, the support of this procedure is mandatory. Fig. 1 shows a typical sequence of signaling messages between the UE and eNB required in CP.

Prior to the periodic UL data transmission, the UE needs time and frequency synchronization with a cell and it thus decodes primary and secondary synchronization signals (NPSS and NSSS). Next, the UE gets the core cell information from the master information block (MIB) and system information blocks (SIBs). At this point, the UE starts the random access (RA) procedure to begin the communication with the network. After the successful contention resolution of the RA, the UE and eNB reestablish the radio resource control (RRC) connection, and the UE switches to RRC connected state. Next, as part of the $\mathrm{CP}$ procedure, the $\mathrm{UE}$ can receive resource allocations through downlink control information (DCI), and send/receive data from the network over narrowband physical uplink shared channel (NPUSCH)/narrowband physical downlink shared channel (NPDSCH). The CP procedure uses RRC 
TABLE II

Summary of Key Parameters Configured in the Radio Interface

\begin{tabular}{|c|c|c|c|}
\hline Parameter & Definition & Range of values & Signaled in \\
\hline Repetitions & $\begin{array}{l}\text { Number of repetitions in the time domain. } \\
\text { Each channel can have a different number } \\
\text { of repetitions }\end{array}$ & $\begin{array}{l}\mathrm{UL} \in[1,2,4,8,16,32,64,128] \\
\mathrm{DL} \in[1,2,4,8,16,32,64,128,192, \\
256,384,512,768,1024,1536,2048]\end{array}$ & $\begin{array}{l}\text { NPRACH repetitions: SIB2 } \\
\text { NPUSCH or NPDSCH repetitions: DCI } \\
\text { NPDCCH repetitions for USS }\left(R_{\text {max }}\right) \text { : } \\
\text { RRC Connection Setup } \\
\text { NPDCCH repetitions for CSS Paging } \\
\text { or RA }\left(R_{\text {max }}\right) \text { : SIB2 }\end{array}$ \\
\hline $\begin{array}{l}\text { DCI subframe } \\
\text { repetitions }\end{array}$ & $\begin{array}{l}\text { Number of repetitions of the DCI in the } \\
\text { time domain }\end{array}$ & $\begin{array}{l}\text { The range of values depends on } \\
\text { the NPDCCH repetitions }\left(R_{\max }\right)\end{array}$ & $\begin{array}{l}\text { DCI repetitions for USS: DCI } \\
\text { DCI repetitions for CSS: SIB2 }\end{array}$ \\
\hline Start subframe $(G)$ & $\begin{array}{l}\text { Starting subframe configuration for an } \\
\text { NPDCCH search space }\end{array}$ & $G \in[1.5,2,4,8,16,32,48,64]$ & $\begin{array}{l}\mathrm{G} \text { for CSS: SIB2 } \\
\mathrm{G} \text { for USS: RRC Connection Setup }\end{array}$ \\
\hline$M C S$ & Modulation and Coding Scheme & {$[0,1,2,3,4,5,6,7,8,9,10,11,12]$} & DCI \\
\hline Radio resources & $\begin{array}{l}\text { Number of Subframes (SFs) for DL } \\
\text { and Resource Units (RUs) for UL }\end{array}$ & {$[1,2,3,4,5,6,8,10]$} & DCI \\
\hline
\end{tabular}

downlink (DL) information transfer packets to forward packets to the UE. While the UE is communicating with the eNB, if the last RRC DL information transfer packet has not been acknowledged at the radio link control (RLC) layer, the UE requests resources to send the confirmation when the periodic buffer status report (BSR) timer expires, starting a scheduling request $(\mathrm{SCH})$ procedure. Later, if the eNB detects an inactivity period greater than the defined RRC Inactivity timer, the eNB initiates the RRC Release procedure to switch the UE to RRC Idle. To save battery, after a period of discontinuous narrowband physical downlink control channel (NPDCCH) monitoring, the UE moves to power saving mode (PSM).

As a summary of the parameters studied in this paper, Table II illustrates key parameters that define the configuration of the communication between the UE and eNB and where these parameters are signaled. For simplicity, we assume the configuration of common search space (CSS) and UE-specific search space (USS) is equal.

\section{POWER, SYNCHRONIZATION, AND OTHER CONSIDERATIONS}

The key of the analytical model is to define the duration of the transmissions/receptions that will significantly impact the energy consumption. To achieve that, this section presents the main considerations underlining in the model.

\section{A. Power Saving Features}

To prolong battery lifetime, NB-IoT extends the value range of two power saving techniques, i.e., extended/enhanced discontinuous reception (eDRX) and PSM. Both techniques enable the UE to enter a power saving state, where it is not required to monitor for paging/scheduling information.

All DRX mechanisms define a cycle, where the UE monitors the DL signaling during a short period of time and sleeps the remaining time of the cycle. This feature can be used while the UE has an active RRC connection with the network (RRC connected state), named as connected-mode DRX (C-DRX), or when there is no RRC connection (RRC Idle state), named as idle-mode DRX (I-DRX).

For simplicity, the model assumes C-DRX is only used after the UE ends its communication with the eNB (i.e., after packet 21 in Fig. 1). When using C-DRX, after expiry of
DRX inactivity time $T_{\mathrm{DRXi}}$, the UE repeatedly starts a C-DRX cycle. This will happen until the connection is released with a RRC Release procedure due to the expiration of the RRC Inactivity timer $T_{\text {inactivity }}$ configured in the network. A C-DRX cycle involves an active listening period $T_{o n D}$, and an inactive period $T_{L C}$. Therefore, the number of C-DRX cycles can be appproximated as $N_{\text {cycles }}^{\mathrm{CDRX}}=\left[\left(T_{\text {inactivity }}-T_{\mathrm{DRXi}}\right) / T_{L C}\right]$, where [] denotes the nearest integer function.

When the UE is in RRC Idle state, the Active Timer (T3324) $T_{\text {active }}$ controls the period the UE is reachable by the network. During this period, there are a number of eDRX cycles. The number of eDRX can be estimated as $N_{\text {cycles }}^{\mathrm{eDRX}}=$ [ $\left.T_{\text {active }} / T_{\mathrm{eDRX}}\right]$, where $T_{\mathrm{eDRX}}$ is the duration of the eDRX. Each eDRX cycle has an active phase controlled by the paging time window (PTW) timer $T_{\mathrm{PTW}}$, and a sleep phase for the remaining period. Withing the PTW, there are several I-DRX cycles (or paging cycles) that can be estimated as $N_{\text {cycles }}^{\mathrm{IDRX}}=$ $\left[T_{\mathrm{PTW}} / T_{P C}\right]$, where $T_{P C}$ is the duration of the I-DRX cycles.

After the expiration of the $T_{\text {active }}$, the UE enters PSM. In PSM, the energy consumption is similar to the power-off state. The UE is not reachable, but it is still registered with the network. The UE exits PSM if there is UL data to send or the UE has to perform a periodic tracking area update (TAU) at the expiration of T3412. To ease the analysis, we do not consider the TAU procedure in the model. Therefore, we assume the UE always exits PSM due to the generation of a new UL report.

\section{B. Power Analysis}

To model the energy consumption of the UE, we assume its behavior can be described as shown in Fig. 2. The model defines five UE power levels.

1) Transmission $\left(P_{T X}\right)$ : The UE is active transmitting a packet to the network, i.e., the TX branch of the UE is on. To obtain the power used by the UE when transmitting, we use the 3GPP's power control equations of the different UL physical channels (see [10]). Hence, $P_{\mathrm{TX}}^{\mathrm{RA}}$ denotes the transmission power for narrowband physical random access channel (NPRACH), and $P_{\mathrm{TX}}$ for the NPUSCH.

2) Reception $\left(P_{R X}\right)$ : The UE is active receiving information from the network, the RX branch of the UE is on. 


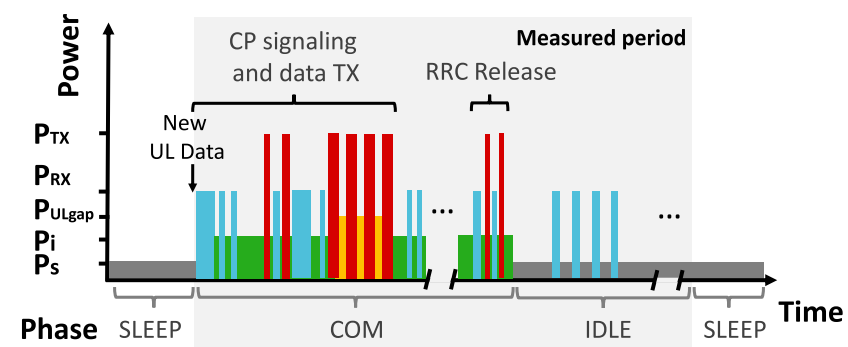

Fig. 2. Example of the considered power levels for the model and the phases in the measurement setup.

3) UL Gap $\left(P_{U L g a p}\right)$ : The UE is active and waiting for the end of the UL transmission gap.

4) Inactive $\left(P_{i}\right)$ : The UE is not transmitting or receiving, thus it is inactive. The accurate clock is ON to maintain the synchronization in the air interface.

5) Standby $\left(P_{S}\right)$ : The UE is in deep sleep low power operation.

The studied UEs will enter standby mode whenever possible (i.e., when the system is quiescent). Then, the $P_{s}$ power level can be seen in PSM as well as during I-DRX inactive periods.

\section{Synchronization}

In order for a UE to connect to the network, it must synchronize with the serving cell. The model considers two different types of synchronization in the analysis.

1) Initial Synchronization: After the UE exits PSM, it needs time and frequency synchronization with the cell.

2) Short Synchronization Before Paging: The UE's standby periods while performing I-DRX cause it has to wake up shortly before the paging occasion to do a short synchronization.

The composition of both synchronization processes will depend on the current coverage of the UE and the cell configuration. To ease their inclusion in the model, we consider a simplified definition. The duration of the initial synchronization depends on three parameters: 1) average required synchronization time $T_{\text {sync }} ; 2$ ) waiting time for the occurrence of the MIB $T_{\mathrm{MIB}-I}$; and 3 ) MIB's reading time $T_{\mathrm{MIB}-\mathrm{RX}}$. The value of $T_{\text {sync }}$ is based on the performance summary found in [11], while the values of $T_{\mathrm{MIB}-I}$ and $T_{\mathrm{MIB}-\mathrm{RX}}$ from [12]. For the short synchronization before paging, its definition is based on the empirical measurements performed in this paper. Then, to estimate the energy consumed, from the measurements we obtain an average power $P_{\mathrm{IDRX}} \mathrm{sync}$ and duration $T_{\text {IDRX }}$ sync .

\section{NPDCCH Scheduling}

The NPDCCH contains the UL and DL scheduling information. Using the NPDCCH, the eNB signals to the UE the specific resources needed in UL or DL through a DCI. There are three formats of DCI: 1) N0, used for UL grant; 2) N1, used for DL scheduling; and 3) N2, used for paging. The possible locations of the NPDCCH carrying DCI are defined by the search spaces. The UE has to monitor these regions within DL Subframes (SFs) to search for DCIs addressed to it. There are two categories of search spaces: 1) the CSS, used for monitoring paging and RA process and 2) the USS, used for monitoring DL or UL UE's specific scheduling information. A set of parameters define the NPDCCH periodicity for each search space [10].

1) $R_{\text {max }}$ : Maximum number of repetitions of NPDCCH.

2) $G$ : Time offset in a search space period.

3) $\alpha_{\text {offset }}$ : Offset of the starting SF in a search period.

4) $T$ : The search space period calculated as $T=R_{\max } \cdot G$ in SF units.

In NB-IoT, the interval between the start of two NPDCCH is referred to as the PDCCH period (pp), thus $\mathrm{pp}=T$. This pp depends on the currently used NPDCCH search space. To consider the periodic occurrence of NPDCCH in the analysis, the waiting time until the next NPDCCH is derived as

$$
T_{W D C}\left(x_{1}, x_{2}, \ldots, x_{n}\right)=\mathrm{pp}-\bmod \left(T_{x_{1}}+T_{x_{2}}+\cdots+T_{x_{n}}, \mathrm{pp}\right)
$$

where $x_{1}, x_{2}, \ldots, x_{n}$ are the considered steps occurred between NPDCCH occasions, $T_{x_{n}}$ is the duration of the $x_{n}$ step, and $\bmod ()$ is the modulus after division function. In this analysis, most of the $x_{n}$ steps between two occurrences of the NPDCCH are: 1) DCI's reception time; 2) wait for the start of NPDSCH/NPUSCH reception/transmission after the end of its associated DCI; and 3) packet reception/transmission time.

Note that to shorten the analysis, $k$ different waiting periods of the NPDCCH will be compacted using the expression $k$. $T_{W D C}\left(x_{1}, x_{2}, \ldots, x_{n}\right)$, although each wait will involve different steps depending on the packet exchange occurred previously.

\section{E. Transmission Gaps}

NB-IoT allows a large set of repetitions to extend coverage. Consequently, the technology also includes transmission gaps. In the DL, the transmission gaps are used to avoid blocking DL resources. In the UL, as a different number of subcarriers can be allocated, this enables simultaneous transmission from several UEs. Then, the blocking of UL resources is not the main reason for UL transmission gaps. These UL transmission gaps are used to allow the UE to resynchronize with the network [13]. The UL gap is defined by a periodicity $T_{\text {GapPeriod }}^{\mathrm{UL}}$ and a gap length $T_{\mathrm{GapDur}}^{\mathrm{UL}}$. Hence, if the duration of the UL transmission is greater or equal than $T_{\text {GapPeriod }}^{\mathrm{UL}}$, the UE applies gaps of $T_{\mathrm{GapDur}}^{\mathrm{UL}}$ with a periodicity $T_{\mathrm{GL} P e r i o d}^{\mathrm{UL}}$ until the transmission is finished. For the DL gap, there are gaps if $R_{\max } \geq N_{\mathrm{GapThr}}^{\mathrm{DL}}$, where $N_{\mathrm{GapThr}}^{\mathrm{DL}}$ denotes the threshold on the maximum number of repetitions. Like UL, the DL gaps are defined by a periodicity $T_{\mathrm{GL} P e r i o d}^{\mathrm{DL}}$ and duration $T_{\mathrm{GapDur}}^{\mathrm{DL}}$.

\section{AnAlytical NB-IOT Model}

In this paper, we provide a Markov chain analysis of the average energy consumed to transfer one UL report using the $\mathrm{CP}$ procedure. The analysis is divided into two different parts. First, we present the Markov chain used to model the behavior of the UE. Then, we estimate the average energy consumption required to perform the $\mathrm{CP}$ procedure. This estimation relies on the stationary probabilities of the Markov chain and the average energy consumption of each Markov chain state. 


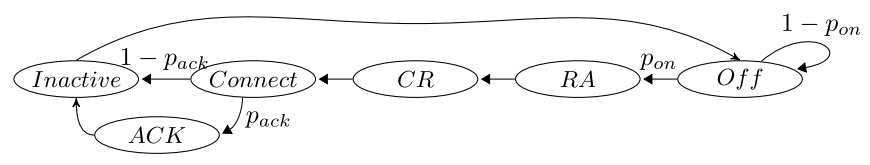

Fig. 3. Markov Chain model for an NB-IoT's UE.

\section{A. Markov Chain Analysis}

Fig. 3 depicts the proposed Markov chain used to model the UE's behavior. This chain is based on the ones used for LTE in [8] and originally proposed in [14] but excluding some aspects for simplicity reasons. Here, we omit the cell capacity limit in the chain. Additionally, the chain does not consider RA failures and access barring. Fig. 1 shows the steps considered at each state of the Markov chain. The states and their transitions are defined as follows.

1) State Off: This state models the situation in which the UE has no new UL report to transmit. In this state, the $\mathrm{UE}$ is using PSM. The UE changes to the $R A$ state when a new UL report is generated.

2) State $R A$ : The $R A$ state represents the synchronization and transmission of the RA preamble. This transmission triggers the transition to the $C R$ state.

3) State $C R$ : This state comprises the request for the RRC connection. After the reception of the RA response (RAR) and later transmission of the RRC request messages, the UE transfers to the connect state.

4) State Connect: This state models the establishment of the RRC connection, and the end of the $\mathrm{CP}$ procedure (including the RLC ACK mode (AM) ACK of the last RRC DL information transfer). After the completion of the $\mathrm{CP}$ procedure, the UE transfers to the $A C K$ state if there is a pending DL response from the IoT server, otherwise, it transfers to the inactive state.

5) State ACK: This state represents the reception of the DL response from the IoT server. After this reception, the UE transfers to the inactive state.

6) State Inactive: This state models the period the UE is still reachable by the network before entering PSM, i.e., RRC Inactivity timer period using C-DRX, reception of the RRC Release, the transmission of its RLC AM ACK, and the Active Timer period using I-DRX. At the expiration of the Active Timer, the UE transfers to the Off state.

We assume the traffic is Poisson distributed with rate $\lambda_{\text {app }}$ packets per ms. The UE's data rate is derived from its average IAT in ms, therefore $\lambda_{\text {app }}=1 /$ IAT. Let $p_{\text {on }}$ denote the probability of having UL traffic in a ms, expressed as $p_{\text {on }}=1-e^{-\lambda_{\text {app }}}$. As we assume there is no DL response from the IoT server, $p_{a c k}=0$.

Denote $b_{j}$ as the steady state probability that a UE is at $j$ state. Then, the stationary probability for each state can be derived as

$$
\begin{aligned}
b_{\mathrm{RA}} & =p_{\text {on }} \cdot b_{\text {off }} \\
b_{\mathrm{CR}} & =b_{\mathrm{RA}}=p_{\text {on }} \cdot b_{\text {off }} \\
b_{\text {Connect }} & =b_{\mathrm{CR}}=p_{\text {on }} \cdot b_{\text {off }}
\end{aligned}
$$

$$
\begin{aligned}
b_{\mathrm{ACK}} & =p_{a c k} \cdot b_{\text {Connect }}=p_{a c k} \cdot b_{\mathrm{RA}} \\
b_{\text {Inactive }} & =b_{\text {Connect }}=p_{\text {on }} \cdot b_{\text {off }} .
\end{aligned}
$$

By imposing the probability normalization condition, we obtain $b_{\text {off }}$ as

$$
b_{\text {off }}=\left(1+p_{\text {on }}\left(4+p_{a c k}\right)\right)^{-1} \text {. }
$$

\section{B. Energy Consumption and Delay Analysis}

Now let us calculate the average energy consumption when performing the $\mathrm{CP}$ procedure. The energy consumption is based on the average power and duration of each Markov chain state. Table III contains the definition of the parameters used in the following analysis. The analysis is divided into three parts. First, we detail the energy consumption while receiving or transmitting packets or signaling in NB-IoT. Second, we present estimates of the energy consumption per Markov chain state. Finally, the battery lifetime is estimated.

1) Packet Energy Estimation: While performing the CP procedure, the UE transmits/receives different types of messages. In this paper, each type is analyzed as follows.

DCI Allocations: This case happens when receiving an UL grant or a DL assignment. In order to estimate the energy consumption, we first derive the reception time needed for the DCI. Note that other channels and signals are present in the DL SFs. Due to the broadcast information present in the NB-IoT frame, approximately only 14 out of 20 SFs are available for control and data transmissions. This is a rough assumption based on the broadcast information comes with a low period. Considering this limitation, the DCI reception time $T_{\mathrm{RX}}(d c i)$ in $\mathrm{ms}$ is calculated as follows:

$$
T_{\mathrm{RX}}(d c i)=\left\lceil N_{\mathrm{REP}_{d c i}} \cdot\left(\frac{20}{14}-1\right)\right\rceil+N_{\mathrm{REP}_{d c i}}
$$

where $N_{\mathrm{REP}_{d c i}}$ is the number of DCI repetitions. Due to the DL $\mathrm{SF}$ duration is $1 \mathrm{~ms}$ and we assume each DCI copy requires a whole DL SF, the number of DCI repetitions equals the duration of the DCI. If $R_{\max }>N_{\mathrm{GapTh}}^{\mathrm{DL}}$ there will be DL gaps in the reception. The total duration of the gaps $T_{\mathrm{Gap}}^{\mathrm{DL}}(d c i)$ is derived as

$$
T_{\mathrm{Gap}}^{\mathrm{DL}}(d c i)=\left\lfloor\frac{N_{\mathrm{REP}_{d c i}}}{T_{\mathrm{GapPeriod}}^{\mathrm{DL}}-T_{\mathrm{GapDur}}^{\mathrm{DL}}}\right\rfloor \cdot T_{\mathrm{GapDur}}^{\mathrm{DL}} \cdot
$$

Finally, we can estimate the DCI's energy consumption $E_{r x}(d c i)$ as

$$
E_{r x}(d c i)=P_{\mathrm{RX}} \cdot T_{\mathrm{RX}}(d c i)+P_{i} \cdot T_{\mathrm{Gap}}^{\mathrm{DL}}(d c i) .
$$

UL Packet: The estimated transmission time for packet $x$ is

$$
\begin{aligned}
T_{\mathrm{TX}}(x) & =N_{\mathrm{REP}} \cdot N_{R U} \cdot T_{R U} \cdot N_{\mathrm{seg}}(x) \\
N_{\mathrm{seg}}(x) & =\left\lceil\frac{L_{x}}{T B S\left(M C S, N_{R U}\right)-H_{R L C M A C}}\right\rceil
\end{aligned}
$$

where $N_{\mathrm{REP}}, N_{R U}$, and $N_{\mathrm{seg}}(x)$ are the number of repetitions, resource units (RUs), and segments, respectively. $T_{R U}$ is the duration in ms of the RU, $L_{x}$ is the size of the packet $x$ in bits, $T B S$ is the transport block size for the NPUSCH resulting from the selection of $M C S$ and $N_{R U}$, and $H_{R L C M A C}$ is the size of 
the RLC/MAC headers. Using $T_{\mathrm{TX}}(x)$, the total duration of the UL gaps is derived

$$
T_{\mathrm{Gap}}^{\mathrm{UL}}(x)=\left\lfloor\frac{T_{\mathrm{TX}}(x)}{T_{\mathrm{GapPeriod}}^{\mathrm{UL}}-T_{\mathrm{GapDur}}^{\mathrm{UL}}}\right\rfloor \cdot T_{\mathrm{GapDur}}^{\mathrm{UL}} .
$$

Next, if $N_{\text {seg }}(x)>0$, we estimate the energy consumed due to the reception of DCIs between the packet segments as

$$
\begin{aligned}
E_{\mathrm{seg}}(x)= & \left(N_{\mathrm{seg}}(x)-1\right) \\
& \times\left(P_{\mathrm{RX}} \cdot T_{\mathrm{RX}}(d c i)+P_{i} \cdot\left(T_{W D C}\left(x_{1}, x_{2}, \ldots, x_{n}\right)\right.\right. \\
& \left.\left.\quad+T_{w D C 2 U S}\right)\right)
\end{aligned}
$$

where the steps of $T_{W D C}$ depend on the last transfer, as explained in Section III-D. Finally, the estimated energy consumption due to the packet $x$ transmission is

$$
E_{\mathrm{tx}}(x)=P_{\mathrm{TX}} \cdot T_{\mathrm{TX}}(x)+P_{U L \mathrm{gap}} \cdot T_{\mathrm{Gap}}^{\mathrm{UL}}(x)+E_{\mathrm{seg}}(x)
$$

The UL packets considered in this paper are (sizes in bytes given in parenthesis): 1) req: RRC connection request (9B); 2) scr: Scheduling Request (9B); 3) setCmp: RRC setup complete together with the piggybacked IP UL report (108B); and 4) $r l_{\mathrm{ACK}}$ : RLC AM ACK (2B). Note that $r e q$ and $s c r$ are scheduled with the UL grant contained in the RAR message. Thus, the estimation of the energy consumption of these packets is similar to the others except for the fixed allocation of resources that forces the following configuration: $N_{R U}=4$, $N_{\mathrm{REP}}=1$, and $T B S=88$ bits.

DL Packet: The estimation in this case is similar to the UL packet. The reception time needed for the packet $y$ is

$$
\begin{aligned}
T_{\mathrm{RX}}(y)= & \left\lceil N_{\mathrm{REP}} \cdot N_{S F} \cdot N_{\mathrm{seg}}(y) \cdot\left(\frac{20}{14}-1\right)\right\rceil \\
& +N_{\mathrm{REP}} \cdot N_{S F} \cdot N_{\mathrm{seg}}(y) \\
N_{\mathrm{seg}}(y)= & \left\lceil\frac{L_{y}}{T B S\left(M C S, N_{S F}\right)-H_{\text {RLCMAC }}}\right\rceil
\end{aligned}
$$

where $N_{S F}$ is the number of SFs, and TBS is the TBS for the NPDSCH resulting from the selection of $M C S$ and $N_{S F}$. Due to the DL SF duration is $1 \mathrm{~ms}$, the total number of DL resources for the reception [i.e., $N_{\mathrm{REP}} \cdot N_{S F} \cdot N_{\mathrm{seg}}(y)$ ] equals the duration of the reception. If $R_{\max }>N_{\mathrm{GapTh}}^{D L}$ there will be DL gaps in the reception. Additionally, if $N_{\text {seg }}(y)>0$, we need to include the reception of the DCIs between segments. Therefore, both effects are estimated as

$$
\begin{aligned}
T_{\mathrm{Gap}}^{D L}(y)= & \left\lfloor\frac{N_{\mathrm{REP}} \cdot N_{S F} \cdot N_{\mathrm{seg}}(y)}{T_{\mathrm{GapPeriod}}^{D L}-T_{\mathrm{GapDur}}^{D L}}\right\rfloor \cdot T_{\mathrm{GapDur}}^{D L} \\
E_{\mathrm{seg}}(y)= & \left(N_{\mathrm{seg}}(y)-1\right) \\
& \quad \times\left(P_{\mathrm{RX}} \cdot T_{\mathrm{RX}}(d c i)+P_{i} \cdot\left(T_{W D C}\left(y_{1}, y_{2}, \ldots, y_{n}\right)\right.\right. \\
& \left.\left.\quad+T_{w D C 2 D S}\right)\right) .
\end{aligned}
$$

Finally, the estimated energy consumption due to the reception of the packet $y$ is

$$
E_{r x}(y)=P_{\mathrm{RX}} \cdot T_{\mathrm{RX}}(y)+P_{i} \cdot T_{\mathrm{Gap}}^{D L}(y)+E_{\mathrm{seg}}(y) .
$$

The DL packets considered in this paper are (sizes in bytes given in parenthesis): 1) rar: RAR (32B); 2) set: RRC connection setup (10B); 3) accept: Non-access stratum (NAS) service accept (15B); and 4) rel: RRC Release (2B).
UL HARQ ACKs: This is a special case as ACKs are sent using NPUSCH format 2. While using this format, the RU is always composed of one subcarrier with a length of 4 slots. Therefore, the energy consumption due to the transmission of a $H A R Q_{a c k}$ can be derived as

$$
\begin{aligned}
T_{T X F 2}\left(H A R Q_{a c k}\right)= & N_{\mathrm{REP}} \cdot T_{R U} \\
T_{\mathrm{Gap}}^{\mathrm{UL}}\left(H A R Q_{a c k}\right)= & \left\lfloor\frac{T_{T X F 2}\left(H A R Q_{a c k}\right)}{T_{\mathrm{GapPeriod}}^{\mathrm{UL}}-T_{\mathrm{GapDur}}^{\mathrm{UL}}}\right\rfloor \cdot T_{\mathrm{GapDur}}^{\mathrm{UL}} \\
E_{t x}\left(H A R Q_{a c k}\right)= & P_{\mathrm{TX}} \cdot T_{T X F 2}\left(H A R Q_{a c k}\right) \\
& +P_{U L \mathrm{gap}} \cdot T_{\mathrm{Gap}}^{\mathrm{UL}}\left(H A R Q_{a c k}\right) .
\end{aligned}
$$

2) Energy Consumption Per Markov Chain State: Let $E_{j}$ and $D_{j}$ be the average energy consumption and delay of the $j$ state, respectively. The following equations describe the energy consumption. Note the delay can be estimated by removing the power components $(P)$ of the equations. Then, $E_{j}$ can be estimated as follows.

1) Off State: The UE does not transmit UL packet in current SF $E_{\text {off }}=P_{s} \cdot 1$.

2) RA State: The UE synchronizes and starts the RA procedure

$$
\begin{aligned}
E_{\mathrm{RA}}= & P_{i} \cdot\left(T_{\mathrm{MIB}-I}+T_{\mathrm{RAPeriod}} / 2+T_{\mathrm{Gap}}^{\mathrm{RA}}\right) \\
& +P_{\mathrm{RX}} \cdot\left(T_{\mathrm{sync}}+T_{\mathrm{MIB}-\mathrm{RX}}\right)+P_{\mathrm{TX}}^{\mathrm{RA}} \cdot N_{\mathrm{REP}}^{\mathrm{RA}} \cdot T_{\mathrm{PRE}}
\end{aligned}
$$

where $T_{\text {RAPeriod }} / 2$ denotes the average waiting time for NPRACH resource occurrence.

3) CR State: The UE performs a connection request

$$
\begin{aligned}
E_{\mathrm{CR}}= & P_{i} \cdot\left(\mathrm{pp} / 2+T_{w D C 2 D S}+T_{w D C 2 U S}\right)+E_{r x}(d c i) \\
& +E_{r x}(\text { rar })+E_{t x}(r e q) .
\end{aligned}
$$

4) Connect State: After a successful connection, the UE sends its data packet. For the CP procedure setup, the data is transmitted piggybacked in the RRC connection setup complete message

$$
\begin{aligned}
E_{\text {Connect }}= & P_{i}\left(2 T_{w D C 2 D S}+T_{w D C 2 U S}+2 T_{\mathrm{ACK}-k 0}\right. \\
& \left.+3 T_{W D C}\left(x_{1}, x_{2}, \ldots, x_{n}\right)\right)+3 E_{r x}(d c i) \\
& +E_{r x}(\text { set })+2 E_{t x}\left(\text { HARQ } Q_{a c k}\right)+E_{t x}(\text { setCmp }) \\
& +E_{r x}(\text { accept })+\left(1-p_{a c k}\right) E_{\mathrm{schCmp}}
\end{aligned}
$$

where $E_{\text {schCmp }}$ is the energy consumed to perform an $\mathrm{SCH}$ when requesting resources to send an RLC ACK. Then, $E_{\text {schCmp }}$ can be estimated as

$$
\begin{aligned}
E_{\mathrm{schCmp}}= & P_{i} \cdot\left(T_{\mathrm{PeriodBSR}}+T_{\mathrm{RAPeriod}} / 2+\mathrm{pp} / 2\right. \\
& +T_{w D C 2 D S}+2 T_{w D C 2 U S}+T_{\mathrm{GAP}}^{\mathrm{RA}} \\
& \left.+T_{W D C}\left(x_{1}, x_{2}, \ldots, x_{n}\right)\right) \\
& +2 E_{r x}(d c i)+E_{r x}(r a r) \\
& +P_{\mathrm{TX}}^{\mathrm{RA}} \cdot N_{\mathrm{REP}}^{\mathrm{RA}} \cdot T_{\mathrm{PRE}}+E_{t x}(s c r) \\
& +E_{t x}\left(r l c_{\mathrm{ACK}}\right) .
\end{aligned}
$$


5) ACK State: The UE receives the IoT server's DL response

$$
\begin{aligned}
E_{\mathrm{ACK}}= & P_{i}\left(T_{W D C}\left(x_{1}, x_{2}, \ldots, x_{n}\right)+T_{w D C 2 D S}\right. \\
& \left.+T_{\mathrm{ACK}-k 0}\right) \\
& +E_{r x}(d c i)+E_{r x}(\text { DLack })+E_{t x}\left(\text { HARQ } Q_{a c k}\right) \\
& +p_{a c k} \cdot E_{\mathrm{schCmp}}
\end{aligned}
$$

6) Inactive State: The UE stays in this state until the expiration of the Active Timer. This state includes C-DRX, the RRC Release, and I-DRX

$$
\begin{aligned}
& E_{\text {Inactive }}=E_{\mathrm{CDRX}}+P_{i} \\
& \times\left(\mathrm{pp} / 2+T_{w D C 2 D S}+T_{\mathrm{ACK}-k 0}\right. \\
& \left.T_{w I D R X}\right)+E_{r x}(r e l)+E_{t x}\left(H A R Q_{a c k}\right) \\
& +E_{\text {schRel }}+E_{\text {IDRX }} \\
& E_{\mathrm{CDRX}}=P_{i}\left(N_{\text {cycles }}^{\mathrm{CDRX}} \cdot\left(T_{L C}-T_{\text {ond }}\right)\right) \\
& +P_{\mathrm{RX}} \cdot\left(T_{\mathrm{DRXi}}+N_{\mathrm{cycles}}^{\mathrm{CDRX}} \cdot T_{o n D}\right) \\
& E_{\mathrm{IDRX}}=N_{\text {cycles }}^{\mathrm{eDRX}}\left(P_{s} \cdot\left(T_{\mathrm{eDRX}}-T_{\mathrm{PTW}}\right)\right. \\
& +N_{\text {cycles }}^{\mathrm{IDRX}}\left(P_{s}\left(T_{P C}-\left(N_{\mathrm{REP}_{d c i}}+T_{\mathrm{IDRX}_{\mathrm{sync}}}\right)\right)\right. \\
& +P_{\mathrm{RX}} \cdot N_{\mathrm{REP}_{d c i}} \\
& \left.\left.+P_{\mathrm{IDRX}_{\mathrm{sync}}} \cdot T_{\mathrm{IDRX}_{\mathrm{sync}}}\right)\right) \\
& +P_{s} \cdot\left(T_{\text {active }}-N_{\text {cycles }}^{\mathrm{eDRX}} \cdot T_{\mathrm{eDRX}}\right)
\end{aligned}
$$

where $E_{\text {schRel }}$ is the energy consumed when requesting resources using the $\mathrm{SCH}$ procedure after the RRC Release. Unlike $E_{\text {schCmp }}, E_{\text {schRel }}$ does not include the complete $\mathrm{SCH}$ procedure, only up to the request of resources (i.e., packets $25-28$ in Fig. 1). This definition of $E_{\text {schRel }}$ is included in the model to emulate the behavior seen in the experimental measurements with the evaluated DUTs. Thus, $E_{\text {schRel }}$ is estimated as

$$
\begin{aligned}
E_{\text {schRel }}= & P_{i} \cdot\left(T_{\text {PeriodBSR }}+T_{\text {RAPeriod }} / 2+\mathrm{pp} / 2\right. \\
& \left.+T_{w D C 2 D S}+T_{w D C 2 U S}+T_{\mathrm{GAP}}^{\mathrm{RA}}\right)+E_{r x}(d c i) \\
& +E_{r x}(\text { rar })+P_{\mathrm{TX}}^{\mathrm{RA}} \cdot N_{\mathrm{REP}}^{\mathrm{RA}} \cdot T_{\mathrm{PRE}}+E_{r x}(s c r) .
\end{aligned}
$$

\begin{tabular}{|c|c|c|c|}
\hline & Parameter & Value & Description \\
\hline \multirow{3}{*}{$\begin{array}{l}\text { 愈 } \\
\text { 离 }\end{array}$} & $E_{j}$ & Variable & Average energy consumption in state $j(\mu \mathrm{J})$ \\
\hline & $E(x)$ & Variable & Packet $x$ average energy consumption $(\mu \mathrm{J})$ \\
\hline & $P_{l}$ & Variable & Average power consumption in $\mathrm{mW}$ for \\
\hline \multirow{7}{*}{ 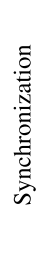 } & & & Average power consumption while \\
\hline & $P_{I D R X_{s y n c}}$ & B: 65.6 & performing short synchronizations in \\
\hline & & & 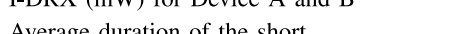 \\
\hline & $T_{I D R X_{s y n c}}$ & 250 & synchronizations during I-DRX (ms) \\
\hline & $T_{\text {sync }}$ & 547.5 & Average initial synchronization time (ms) \\
\hline & $T_{M I B-I}$ & 103 & MIB waiting time $(\mathrm{ms})$ \\
\hline & $T_{M I B-R X}$ & 8 & MIB reception time (ms) \\
\hline \multirow{3}{*}{$\overleftrightarrow{\nwarrow}$} & $T_{R A P e r i o d}$ & 640 & NPRACH periodicity (ms) \\
\hline & $T_{P R E}$ & 5.6 & Preamble format 0 duration $(\mathrm{ms})$ \\
\hline & $N_{R E P}^{R A}$ & Variable & Number of preamble repetitions \\
\hline \multirow{5}{*}{ 气ै } & & & NPRACH gap duration (ms) \\
\hline & $T_{G a p}^{R A}$ & 0,40 & $\begin{array}{l}\text { If NPRACH repetitions }>64 \text {, } \\
T^{R A}=40 \text { otherwise } T^{R A}=0\end{array}$ \\
\hline & $T_{\text {GapPeriod }}^{s}$ & 296,128 & $\begin{array}{l}\text { Gap } \\
\text { Gap periodicity for UL and DL (ms) }\end{array}$ \\
\hline & $T_{\text {GapDur }}^{s}$ & 40,32 & Gap duration for UL and DL (ms) \\
\hline & $N_{G a p T h r}^{D L}$ & 64 & DL gap threshold \\
\hline \multirow{4}{*}{ 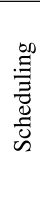 } & $T_{w D C 2 U S}$ & 8 & $\begin{array}{l}\text { Start of NPUSCH transmission after } \\
\text { the end of its associated DCI (ms) }\end{array}$ \\
\hline & $T_{w D C 2 D S}$ & 5 & $\begin{array}{l}\text { Start of NPDSCH transmission after } \\
\text { the end of its associated DCI (ms) }\end{array}$ \\
\hline & $T_{A C K-k 0}$ & 13 & Delay for the ACK of a DL packet (ms) \\
\hline & $T_{\text {PeriodBSR }}$ & 8 & Buffer Status Report (BSR) Timer ( $p p)$ \\
\hline \multirow{11}{*}{ 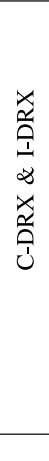 } & $T_{D R X i}$ & 2 & Period the UE should remain monitoring \\
\hline & $T_{\text {inactivity }}$ & 20 & RRC Inactivity timer (s) \\
\hline & $T_{o n D}$ & Variable & On duration timer during a C-DRX cycle \\
\hline & $N p p_{o n D}$ & 8 & Number of consecutive NPDCCH periods \\
\hline & $T_{L C}$ & 2.048 & C-DRX Long Cycle (s) \\
\hline & $T_{w I D R X}$ & 1.1 & Wait before entering I-DRX after \\
\hline & $T_{P C}$ & 2.56 & I-DRX Paging Cycle (s) \\
\hline & $T_{P T W}$ & 20.48 & PTW cycle duration (s) \\
\hline & $T_{e D R X}$ & 81.92 & eDRX cycle duration (s) \\
\hline & Tactive & 120 & Active Timer duration (s) \\
\hline & $H_{R L C M A C}$ & 4 & RLC/MAC headers size (B) \\
\hline
\end{tabular}

Additionally, $T_{o n D}$ specifies the number of consecutive NPDCCH SFs at the beginning of a C-DRX cycle to monitor. This timer is given in units of pp. However, as the duration of the C-DRX cycle could be smaller than the duration of the $T_{o n D}$ due to a large value of $\mathrm{pp}$, the duration of $T_{o n D}$ is estimated as

$$
T_{\text {onD }}=\min \left(\left[T_{L C} / \mathrm{pp}\right], N p p_{\text {onD }}\right) \cdot R_{\max }^{U S S}
$$

where $N p p_{o n D}$ is the number of pp defined at the onDuration Timer, and $R_{\max }^{U S S}$ is the maximum number of repetitions for NPDCCH for USS.

3) Battery Lifetime Estimation: From the prior analysis the energy consumed per day $E_{\text {day }}^{\text {model }}$ in joules (J) is estimated as

$$
E_{\text {day }}^{\text {model }}=\left(\left(\sum_{j} b_{j} E_{j}\right) \cdot \frac{D_{\text {day }}}{\sum_{j} b_{j} D_{j}}\right) \cdot 1 e-6
$$

TABLE III

VARIABles AND PARAMETERS OF THE Model

where $D_{\text {day }}$ denotes the duration of one day. Finally, the battery lifetime in years $Y_{\text {model }}$ can be estimated as

$$
\begin{aligned}
E_{\text {day }_{W h}}^{\text {model }} & =\frac{E_{\text {day }}^{\text {model }}}{3600} \\
Y_{\text {model }} & =\frac{C_{\text {bat }}}{E_{\text {dad }_{W h}}^{\text {model }_{W}} \cdot 365.25}
\end{aligned}
$$

where $E_{\text {day }_{W h}}^{\text {model }}$ is the energy consumption per day in watt-hour units, and $C_{\text {bat }}$ is the battery capacity.

\section{EXPERIMENTAL METHOdOLOGY}

To validate the NB-IoT model, we use our experimental setup to measure the UE's energy consumption while sending an UL report using CP procedure. To do that, the DUT will be connected to a base station while the voltage and current draw are measured. In this paper, we use a Keysight E7515A UXM wireless test set (UXM) as an NB-IoT base station emulator. The UXM supports NB-IoT's Release 13, and this is also the release assumed in this paper. Fig. 4 shows the measurement 


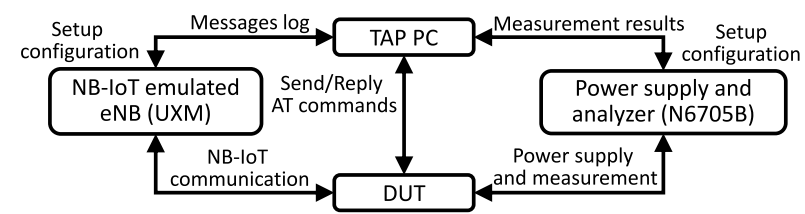

Fig. 4. Measurement setup.

setup, based on the experimental setup used in [7]. The DUT is wired through its antenna port to the UXM. Additionally, the DUT is powered with a Keysight N6705B dc power analyzer. The N6705B acts as a power supply and measures the voltage and current draw by the DUT. Finally, Keysights test automation platform (TAP) is used to control the different elements of the experimental setup. The TAP provides the following utilities: 1) communication with the DUT through AT commands; 2) an unified interface to configure the UXM and N6705B; and 3 ) synchronization of the protocol logs and measurements with $\leq 1 \mathrm{~ms}$ accuracy. Two commercial NB-IoT UEs are used as DUTs. The UEs run firmware from August 2018.

The validation of the model is done based on three test cases. These test cases address different main parts of the proposed model.

1) G: This test focuses on the evaluation of the $G$ parameter. The value of $G$ together with $R_{\max }$ defines pp (Section III-D). Then, the general scheduling process.

2) REP: This test is a simplification of the use of repetitions as all the parameters related to repetitions are set equal. The goal is to examine the energy consumption impact due to an increase of repetitions to extend coverage.

3) SCS: Considering the two subcarrier spacing (SCS) allowed in NB-IoT (15 and $3.75 \mathrm{kHz}$ ). This test compares the performance of both single subcarrier configurations. If the UE has not reached its maximum transmission power, following the power control mechanism, ideally, the decrease of $S C S$ will increase the duration of the $R U$ and decrease the transmission power equally.

Each point of the experimental evaluation showed in this paper is based on one empirical realization. Table IV shows the baseline configuration of the radio interface between the DUT and the UXM. Table V summarizes the specific UXM settings for each test case considered. The configuration of the parameters chosen forces the maximum transmission power $p_{\max }$, except for the SCS test case. For SCS, the power control is configured to use $p_{\max }$ when $S C S=15 \mathrm{kHz}$, and reduce the power as obtained from the power control mechanism when reducing the $S C S$ to $3.75 \mathrm{kHz}$ [10]. For all test cases, the N6705B sampling time is $1 \mathrm{~ms}$, and the current range is set to auto. This enables to automatically change the measurement range during the measurements. Note that unlike the live NBIoT network, the configuration of the data channels NPDSCH and NPUSCH is fixed in the UXM. This means that different packet sizes would be transmitted/received using the same configuration and relying in segmentation if it is needed.

In the tests, the DUT power consumption is measured from the beginning of the CP to the start of PSM. Fig. 2 shows a
TABLE IV

Baseline Configuration of the Main Parameters

\begin{tabular}{|c|c|c|}
\hline & Parameter & Value \\
\hline$\overline{0}$ & $\begin{array}{l}p_{\max } \\
\mathrm{RSRP} \\
\text { Reference Signal indicator } \\
\alpha \\
P 0_{\text {nominal }} \\
P 0_{U \text { Especific }} \\
\text { Preamble initial power }\end{array}$ & $\begin{array}{c}23 \mathrm{dBm} \\
-110 \mathrm{dBm} / 15 \mathrm{kHz} \\
27 \mathrm{dBm} \\
1 \\
-67 \mathrm{dBm} \\
0 \mathrm{dBm} \\
-90 \mathrm{dBm}\end{array}$ \\
\hline 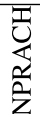 & $\begin{array}{l}\text { Periodicity } \\
\mathrm{N}_{R E P}^{R A} \\
\text { RAR Window Size }\end{array}$ & $\begin{array}{c}640 \mathrm{~ms} \\
2 \\
5 p p\end{array}$ \\
\hline 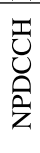 & $\begin{array}{l}R_{\max } \text { for USS and CSS } \\
G \text { for USS and CSS } \\
N_{R E P_{d c i}} \text { for CSS } \\
N_{R E P_{d c i}} \text { for USS }\end{array}$ & $\begin{array}{l}8 \\
2 \\
8 \\
1\end{array}$ \\
\hline 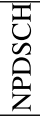 & $\begin{array}{l}\text { MCS } \\
N_{S F} \\
N_{R E P}\end{array}$ & $\begin{array}{c}3 \\
10 \\
1\end{array}$ \\
\hline $\begin{array}{l}\text { U. } \\
\text { Dे } \\
\text { z }\end{array}$ & $\begin{array}{l}\text { MCS } \\
N_{R U} \\
N_{R E P} \\
\text { Number of subcarriers } \\
\text { Subcarrier spacing (SCS) }\end{array}$ & $\begin{array}{c}3 \\
10 \\
1 \\
1 \\
15 \mathrm{kHz}\end{array}$ \\
\hline
\end{tabular}

example of the mentioned measured period. To compare the results of the tests cases with the model, we define three phases during the measurements.

1) COM: UE wakes up, sends its data using $\mathrm{CP}$, monitors NPDCCH while applying C-DRX, and releases the RRC connection after the reception of the RRC Release packet.

2) IDLE: UE stays in I-DRX until $T_{\text {active }}$ expiration.

3) SLEEP: UE sleeps using PSM until the next transmission.

From the measured energy consumption when sending one UL report, we can estimate the energy consumed per day considering a specific IAT. For simplicity, when comparing with the model, we assume the duration of the $T_{\text {SLEEP }}^{\text {test }}=$ IAT. Then, the average energy consumed per day $E_{\text {day }}^{\text {test }}$ and the battery lifetime in years $Y_{\text {test }}$ can be estimated as follows:

$$
\begin{aligned}
N_{\text {reports }_{\text {day }}} & =\frac{D_{\text {day }}}{T_{\mathrm{COM}}^{\text {test }}+T_{\text {IDLLE }}^{\text {test }}+T_{\text {SLEEP }}^{\text {test }}} \\
E_{\text {day }_{W h}}^{\text {test }} & =\frac{N_{\text {reports }} \cdot\left(E_{\mathrm{COM}}^{\text {test }}+E_{\text {IDLE }}^{\text {test }}+E_{\text {SLEEP }}^{\text {test }}\right)}{3600} \\
Y^{\text {test }} & =\frac{C_{\text {bat }}}{E_{\text {day }_{W h}}^{\text {test }} \cdot 365.25}
\end{aligned}
$$

where $N_{\text {reports }}$ day denotes the number of UL reports sent in one day, $T_{i}^{\text {test }}$ and $E_{i}^{\text {test }}$ are the duration and energy consumed in the $i$ th phase, respectively. As mentioned before, the SLEEP phase is not measured in the experimental setup. Then, the energy consumed in this phase is estimated as $E_{\mathrm{SLEEP}}^{\mathrm{test}}=P_{s} \cdot T_{\mathrm{SLEEP}}^{\mathrm{test}}$. 
TABLE $\mathrm{V}$

Test Cases With UXM Settings

\begin{tabular}{|c|c|c|}
\hline Test case & Sweeping parameter & Other settings \\
\hline G & $G=\{1.5,2,4,8,16,32,48,64\}$ & \\
\hline REP & $\begin{array}{l}R_{\max }=\{1,2,4,8,16,32,64\} \\
N_{R E P_{d c i}}=N_{R E P}=R_{\max }\end{array}$ & $\begin{array}{l}G=\{8,4,2,2,2,2,2\} \\
\text { CSS and USS are set equal }\end{array}$ \\
\hline SCS & $S C S=\{3.75,15\} \mathrm{kHz}$ & $\begin{array}{l}P 0_{\text {nominal }}=-68 \mathrm{dBm} \\
\alpha=0.7 \\
N_{R E P}=2\end{array}$ \\
\hline
\end{tabular}

\section{NUMERICAL RESULTS}

This section contains the validation results of our proposed analytical NB-IoT model using two different DUTs. The validation is done in terms of battery lifetime and latency to perform the CP, two targets of NB-IoT. For both results, we compare the values obtained with the following.

1) The analytical model presented in Section IV, named in the following figures as "model." For the three test cases studied, the analytical model is configured with the same parameter values as the experimental setup.

2) The estimation from the measurements obtained with the experimental setup presented in Section V, named in the figures as "measurements."

We consider the periodic UL reports are UDP packets with $50 \mathrm{~B}$ of payload, and the UE battery capacity is $C_{\text {bat }}=$ 5 Wh [1]. Table VI lists the measured average power consumption levels for the two DUTs evaluated. Fig. 5 shows the battery lifetime obtained for an IAT of $24 \mathrm{~h}$ when using the analytical model and the measurements for the test cases $G$ and REP, respectively. The increase of the parameter $G$ does not have a noticeable impact on the battery lifetime. Although the increase of $G$ delays the scheduling of resources, the UE stays inactive while waiting and therefore the energy consumption increase is small. On the contrary, the increase of the number of repetitions has a significant impact on the battery lifetime. For smaller IATs such as 6 min, the battery lifetime has the same trend with much lower values. For example, in this case, the battery lifetime measurements with device A ranges from 14 weeks to 1 week when the number of repetitions is increased from 1 to 64 . Considering the target battery lifetime of ten years in NB-IoT, the correct use of repetitions to extend coverage, together with the knowledge of the UE traffic profile are essential to achieving it. Note battery lifetime and coverage are conflicting targets, for battery powered smart meters located in remote areas (i.e., agriculture or environmental monitoring applications), these KPIs are critical due to their costly access for maintenance. To extend coverage up to the $164 \mathrm{~dB}$ MCL, repetitions are essential. The resulting battery lifetime when using a more robust configuration based on repetitions may be more optimistic in practical deployments than the results shown in Fig. 5. The reasoning is REP test case assumes all channels have the same number of repetitions. However, in practical deployments, the requirements of each channel are different. Thus, it may entail a distinct configuration of repetitions per channel.

Additionally, for the SCS test case and $S C S=15 \mathrm{kHz}$, both DUTs achieve a similar battery lifetime of average 18 years for

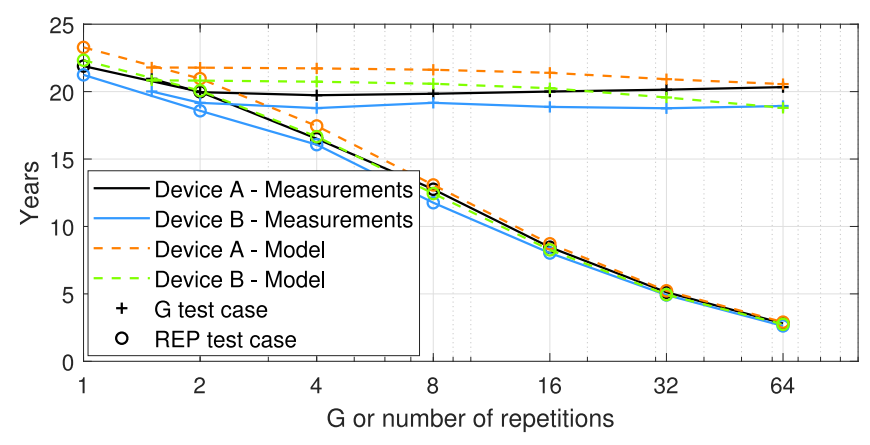

Fig. 5. Battery lifetime estimation as a function of $G$ (G test case) or repetitions (REP test case) for an IAT of $24 \mathrm{~h}$.

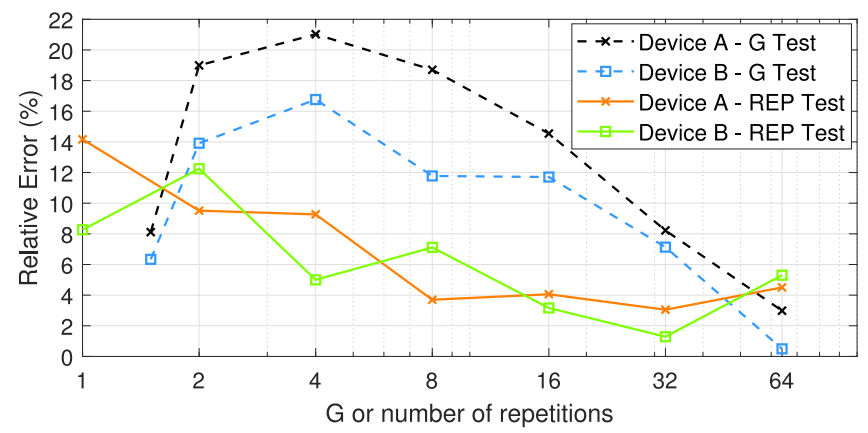

Fig. 6. Relative error of the battery lifetime estimation in years between the analytical model and the measurements assuming an IAT of 6 min.

an IAT of $24 \mathrm{~h}$. However, when reducing the $S C S$ to $3.75 \mathrm{kHz}$, the battery lifetime decreases an average of $20 \%$ as the power consumption decrease and $T_{R U}$ increase are unequal. Despite the $3.75 \mathrm{kHz}$ SCS obtains worse results, it is an interesting configuration for deep indoor IoT scenarios where a large number of UEs are concentrated in a small area and most of them experience a significant penetration loss. The reason is this configuration provides more robust communication with the eNB and enables more simultaneous connections.

To ease the comparison of the analytical model and the measurements, Fig. 6 illustrates the relative error resulting from both estimations of the battery lifetime in years for G and REP test cases, considering the two DUTs and an IAT of 6 min. The maximum relative error obtained between the model and the measurements is $21 \%$. We use the IAT of $6 \mathrm{~min}$ as a pessimistic scenario for periodic reporting. For example, the smallest IAT considered in [1] for periodic mobile autonomous reporting is $30 \mathrm{~min}$. The error decreases as the value of the parameter $G$ or the number of repetitions increases. This is because the energy consumed while performing $\mathrm{CP}$ increases. Therefore, the model estimation improves as the $\mathrm{CP}$ procedure becomes more important than other assumed simplifications. Particularly, the main factors in the relative error are as follows.

1) The simplification of the synchronizations (i.e., the initial synchronization and the short synchronizations before paging) modeling: both power-hungry and robust processes have been modeled with an average duration 
TABLE VI

Measured Average Power Consumption

\begin{tabular}{l|c|c}
\hline & Device A & Device B \\
\hline$P_{T X} \dagger$ at $23 \mathrm{dBm}$ & $731 \mathrm{~mW}$ & $765 \mathrm{~mW}$ \\
\hline$P_{T X} \ddagger$ at $17 \mathrm{dBm}$ & $311 \mathrm{~mW}$ & $503 \mathrm{~mW}$ \\
\hline$P_{\boldsymbol{R} X}$ & $215 \mathrm{~mW}$ & $242 \mathrm{~mW}$ \\
\hline$P_{\boldsymbol{U} L \text { gap }}$ & $128.4 \mathrm{~mW}$ & $160.4 \mathrm{~mW}$ \\
\hline$P_{\boldsymbol{i}}$ & $17.8 \mathrm{~mW}$ & $29.1 \mathrm{~mW}$ \\
\hline$P_{S}$ & $14.14 \mu W$ & $11.13 \mu W$ \\
\hline
\end{tabular}

$\dagger$ TX power for G, REP test cases, and when $S C S=15 \mathrm{kHz}$ in SCS. $\ddagger$ TX power for $S C S=3.75 \mathrm{kHz}$ in SCS test case.

and power consumption. However, both synchronizations entail several steps and their performance depend on channel quality and the NB-IoT deployment [3], [4].

2) The assumed statistical average prior to the transmission of a preamble. In the system model considered, the preamble transmission happens in three different signaling exchanges. We always assume the wait for NPRACH resources to send the preamble is half the NPRACH periodicity (i.e., its statistical average). However, this wait can range from a few ms to the NPRACH period value in the measurements as we only consider one empirical realization per measurement.

For larger IATs than $6 \mathrm{~min}$, the relative error is smaller. For example, the maximum relative error is $11 \%$ when the IAT is $24 \mathrm{~h}$. In this case, the reduction is due to the larger PSM duration that is easily modeled with its average power consumption. Additionally, for SCS test case, the resulting average relative error assuming an IAT of $6 \mathrm{~min}$ is $12 \%$ and $6 \%$ for devices $\mathrm{A}$ and $\mathrm{B}$, respectively.

Note REP test case has a limited evaluation of the repetitions up to 64. This is due to limitations of the testbed. Despite the model is not validated for a higher number of repetitions, there are no reasons to believe the model would not be applicable as it includes the possible segmentation of the packets due to poor radio conditions and the gaps for long transfers.

Finally, Fig. 7 shows the latency to finish the CP procedure for the REP test case. This figure compares the measured latency of both DUTs and the value obtained with the analytical model. As expected, as the number of repetitions increases, the latency is higher. This increment is less notable in other test cases. For example, the maximum latency reached is $10.35 \mathrm{~s}$ for $G=64$ in $\mathrm{G}$ test case, and $4.81 \mathrm{~s}$ for $S C S=3.75 \mathrm{kHz}$ in SCS test case. Note that the difference between the model and the measurements are greater when estimating the CP's latency than the battery lifetime. This is due to the model does not consider retransmissions the UE could experience, the simplification of the synchronization, and some waits of the UEs seen in the measurements but not included in the model.

\section{CONCLUSION}

In this paper, we propose and validate an analytical NB-IoT model. The analytical model estimates the average energy consumption and delay of a UE sending periodic UL reports using $\mathrm{CP}$ optimization. This estimation is based on a 6-state Markov chain. For the validation, an experimental setup consisting of a base station emulator and commercial NB-IoT UEs are used

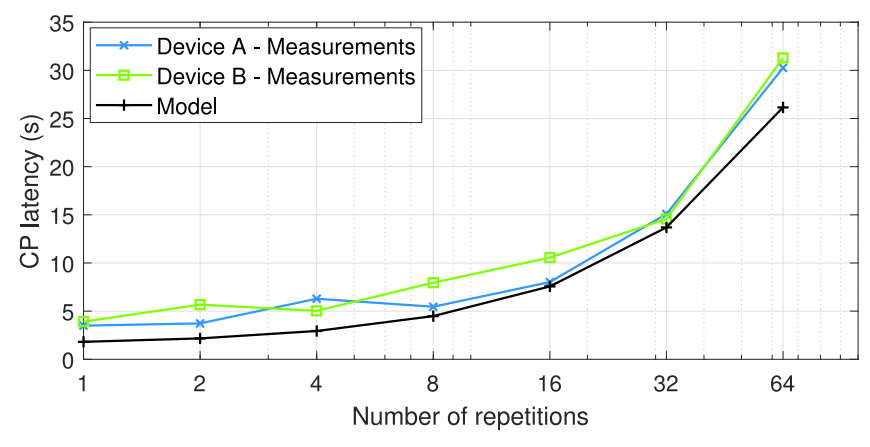

Fig. 7. Comparison of the latency to finish $\mathrm{CP}$ procedure measured in both DUTs and obtained with the analytical model for REP test case.

to measure the energy consumption. The validation is done in terms of UEs battery lifetime and latency to finish the $\mathrm{CP}$ procedure.

The evaluation is done considering two different NB-IoT UEs and three test cases. These test cases address main parts of the analytical model: 1) G: the scheduling process of NB-IoT; 2) REP: the lengthening of transmissions/receptions to extend coverage; and 3) SCS: performance of the single subcarrier configurations. The results show the analytical model performs well, obtaining a maximum relative error of the battery lifetime estimation in years between the model and the measurements of $21 \%$ assuming an IAT of 6 min. Additionally, the results demonstrate the NB-IoT UEs achieve the targets of ten years of battery lifetime or $10 \mathrm{~s}$ of latency for a large range of results when the traffic profile has a large IAT, or the configuration of the radio resources do not require an extensive number of repetitions.

\section{ACKNOWLEDGMENT}

The authors would like to thank Keysight Technologies, Aalborg, Denmark, for helping with the UXM and TAP.

\section{REFERENCES}

[1] "Cellular system support for ultra-low complexity and low throughput Internet of Things (CIoT) (Rel-13), v13.1.0," 3GPP, Sophia Antipolis, France, Rep. TR 45.820, 2015.

[2] R. Ratasuk, N. Mangalvedhe, J. Kaikkonen, and M. Robert, "Data channel design and performance for LTE narrowband IoT," in Proc. IEEE 84th Veh. Technol. Conf. (VTC-Fall), Montreal, QC, Canada, Sep. 2016, pp. 1-5.

[3] A. Adhikary, X. Lin, and Y. P. E. Wang, "Performance evaluation of NB-IoT coverage," in Proc. IEEE 84th Veh. Technol. Conf. (VTC-Fall), Montreal, QC, Canada, Sep. 2016, pp. 1-5.

[4] L. Feltrin et al., "Narrowband IoT: A survey on downlink and uplink perspectives," IEEE Wireless Commun., vol. 26, no. 1, pp. 78-86, Feb. 2019.

[5] A. Azari, G. Miao, C. Stefanovic, and P. Popovski, "Latencyenergy tradeoff based on channel scheduling and repetitions in NBIoT systems," in Proc. IEEE Glob. Commun. Conf. (GLOBECOM), Dec. 2018, pp. 1-7.

[6] M. E. Soussi, P. Zand, F. Pasveer, and G. Dolmans, "Evaluating the performance of eMTC and NB-IoT for smart city applications," in Proc. IEEE Int. Conf. Commun. (ICC), Kansas City, MO, USA, May 2018, pp. $1-7$.

[7] M. Lauridsen, R. Krigslund, M. Rohr, and G. Madueno, "An empirical NB-IoT power consumption model for battery lifetime estimation," in Proc. IEEE 87th Veh. Technol. Conf. (VTC Spring), Porto, Portugal, Jun. 2018, pp. 1-5. 
[8] P. Andres-Maldonado, P. Ameigeiras, J. Prados-Garzon, J. J. Ramos-Munoz, and J. M. Lopez-Soler, "Optimized LTE data transmission procedures for IoT: Device side energy consumption analysis," in Proc. IEEE Int. Conf. Commun. Workshops (ICC Workshops), Paris, France, May 2017, pp. 540-545.

[9] General Packet Radio Service Enhancements for Evolved Universal Terrestrial Radio Access Network Access (Rel-13), v13.13.0, 3GPP Standard TS 23.401, 2017.

[10] Evolved Universal Terrestrial Radio Access; Physical Layer Procedures (Rel-13) V13.11.0, 3GPP Standard TS 36.213, 2018.

[11] "Summary of evaluation results for NB-PSS," 3GPP, Sophia Antipolis, France, Rep. R1-162048, 2016.

[12] "NB LTE-Battery lifetime evaluation," 3GPP, Sophia Antipolis, France, Rep. RP-151393, 2015.

[13] O. Liberg, M. Sundberg, E. Wang, J. Bergman, and J. Sachs, Cellular Internet of Things. London, U.K.: Elsevier, 2018.

[14] G. C. Madueño et al., "Assessment of LTE wireless access for monitoring of energy distribution in the smart grid" IEEE J. Sel. Areas Commun., vol. 34, no. 3, pp. 675-688, Mar. 2016.

Pilar Andres-Maldonado received the B.Sc. degree in telecommunications engineering from the University of Granada, Granada, Spain, in 2015, where she is currently pursuing the Ph.D. degree at the Department of Signal Theory, Telematics and Communications.

From August to November 2018, she was a Visiting Researcher with the Wireless Communication Networks Section, Aalborg University, Aalborg, Denmark, for three months. Her current research interests include machinetype communications, NB-IoT, 5G, and LTE.
Mads Lauridsen received the M.Sc.EE. and Ph.D. degrees in wireless communication from Aalborg University, Aalborg, Denmark, in 2009 and 2015 , respectively.

$\mathrm{He}$ is currently a Wireless Researcher with Nokia Bell Labs, Aalborg. Until 2018, he was with the Wireless Communication Networks Section, Aalborg University. His current research interests include massive machinetype communication, nonterrestrial networks, and UE power saving for $5 \mathrm{G} \mathrm{NR}$

Pablo Ameigeiras received the M.Sc.EE. degree from the University of Malaga, Málaga, Spain, in 1999.

$\mathrm{He}$ performed his master's thesis with the Chair of Communication Networks, RWTH Aachen University, Aachen, Germany. In 2000, he joined Aalborg University, Aalborg, Denmark, where he carried out his Ph.D. thesis. In 2006, he joined the University of Granada, Granada, Spain, where he has been leading several projects in the field of LTE and LTE-Advanced systems. His current research interests include 5G and Internet of Things technologies.

Juan M. Lopez-Soler received the B.Sc. degree in physics (electronics) and the Ph.D. degree in signal processing and communications from the University of Granada, Granada, Spain.

$\mathrm{He}$ is currently a Professor with the Department of Signals, Telematics and Communications, University of Granada. He joined the Institute for Systems Research (formerly, SRC), University of Maryland at College Park, College Park, MD, USA, as a Visiting Faculty Research Assistant. Since its creation in 2012, he has been the Director of the Wireless and Multimedia Networking Laboratory, University of Granada. He has participated in 11 public and 13 private funded research projects and is the coordinator in 14 of them. He has advised six Ph.D. students and has had 24 papers published in indexed journals and contributed to over 40 workshops/conferences. His current research interests include wireless communications, multimedia networking, and real-time middleware. 